\title{
Operative ship monitoring system based on integrating AIS polls within synthetic aperture radar (SAR) imagery
}

\author{
G. Margarit, J. A. Barba \& A. Tabasco \\ URS Division Group, Science and Earth Observation Processing Systems \\ (SEOPS), GMV Space, Spain
}

\begin{abstract}
This paper presents a new ship monitoring system developed at GMV Aerospace that integrates the reports provided by the Automatic Identification System (AIS) with ship-related information derived from SAR data analysis. In contrast to other proposals, SAR data is considered here to be the main input whereas AIS polls the supporting channel. The system kernel is built by the combination of three independent modules (coastline isolation, ship detection and ship classification) with two main purposes: to increase system independence and automatism. The former tries to limit the dependence on ancillary information (such as AIS), whereas the latter on human operator intervention. The three modules are integrated in a common framework developed with state-of-the-art web technologies. The result is a new concept for ship monitoring (including automatic SAR-based ship classification) that helps to better locate the error sources and reduce their dispersion. The system is able to ingest any type of SAR data for different modes and resolution, for instance ERS, ENVISAT, PALSAR, RADARSAT series or TerraSAR-X. Obviously, the performance would be strongly related with sensor features, but the system is designed to let single-polarimetric images with medium resolution provide reasonable results. This adds multi-sensor capability, which helps to reduce report refreshing time. In the paper, some examples will be processed and the main results analyzed. Preliminary tests for the ship classification module will be also presented, profiting from the ground-truth included within AIS-reports.
\end{abstract}




\section{Introduction}

This paper presents an advanced ship monitoring system combining information obtained from transponder polls (such the Automatic Identification System (AIS) [1] from the International Maritime Organization (IMO)) and information inferred from Synthetic Aperture Radar (SAR) data [2]. The work has been seeded from the research carried out in the framework of the MARISS project [3]. There, user interaction (especially police and military authorities) has made it possible to develop a first operative system for supporting decision making. Recently, a second version has been released providing automatic coastline detection and ship classification capabilities.

The justification of this work comes from the necessity of official authorities to control all the activities of ships. Important issues lie in ecosystem protection, providing safety and surveillance along the transportation corridors, illegal immigration monitoring and assuring sustainable economic development. Since the early 1990s, much effort has been devoted to developing monitoring systems based on active on-board transponders [4]. There, GPS-based receivers provide real-time ship status to monitoring centers via satellite communications. However, the experience showed that this approach does not have enough independence to achieve success. Certainly, the uncertainty about whether ship board devices are properly working or, on the contrary, whether the experiment is subject to accidental or intentioned malfunctioning is large. For such a purpose, other alternatives were proposed and SAR is the one gathering more benefits. It can image large scenes with fine resolutions for all-day all-weather monitoring independent from the targets to track.

However, SAR ship monitoring is not evident due to some inherent constraints. The limited resolution, the coherent speckle noise and the image distortions due to the dynamic environment are the greatest problems. All of them have been the main causes for which ship monitoring has been mainly restricted to ship detection of large ships. However, the new generation of SAR sensors, such as TerraSAR-X, Cosmo-Skymed or Radarsat-2, is changing this situation, thanks to the improved resolution, revisiting time and polarimetric capabilities that they provide. With these, the range of targets that can be detected increases to small and less dispersive ships, of special importance for illegal immigration monitoring. In addition, automatic ship classification may be tackled following the seed of recent works, such as [5] [6].

In this framework, this paper presents GMV's SAR-AIS Ship Monitoring (SASHIM), which combines an advanced web interface, developed with stateof-the-art technologies, with a SAR Ship Detection and Classification Algorithm (SIDECAR). It is conceived to drop the dependence on active transponders as much as possible, and to provide a friendly, secure and robust environment for which system performance and outputs can be controlled according to user needs. All the main elements of SASHIM will be described, jointly with some processing examples applied to ENVISAT and ERS data. Cross-correlation of the 
output results with AIS-polls will also be included to show ship detection and classification performance.

\section{System overview}

Figure 1 presents the high-level scheme for the SASHIM system. The four main stages are differentiated, namely:

- Input data streams. This stage automatically ingests input data via dedicated FTP links once new sets are available. Three types of streams are conceived, namely:

1. SAR images with meta-data extraction. Accepted formats are ENVISAT .N1, and ERS .JP2 and .E2. Now, new formats are being included such as TerraSAR-X and ALOS-PALSAR

2. Satellite AIS streams obtained via communication satellite links

3. Coastal AIS streams acquired via coastal on-ground stations.

- Information extraction. This stage is devoted to locating ships from input SAR images by using SIDECAR. There, a multi-scale approach based on wavelets is adopted for locating spots with reflectivity and morphological gradient variance. Before detecting ships, accurate land masking is required. Right now, this is performed by analyzing reflectivity gradient variances with the support of shape coastline .shp files.

- Value Addition. This stage improves data extraction by retrieving additional information. Two issues are mainly covered, namely: 1) the correlation of SAR ship detection with AIS polls and 2) the classification of ships by analyzing the distribution of the Radar Cross Section (RCS) along SAR

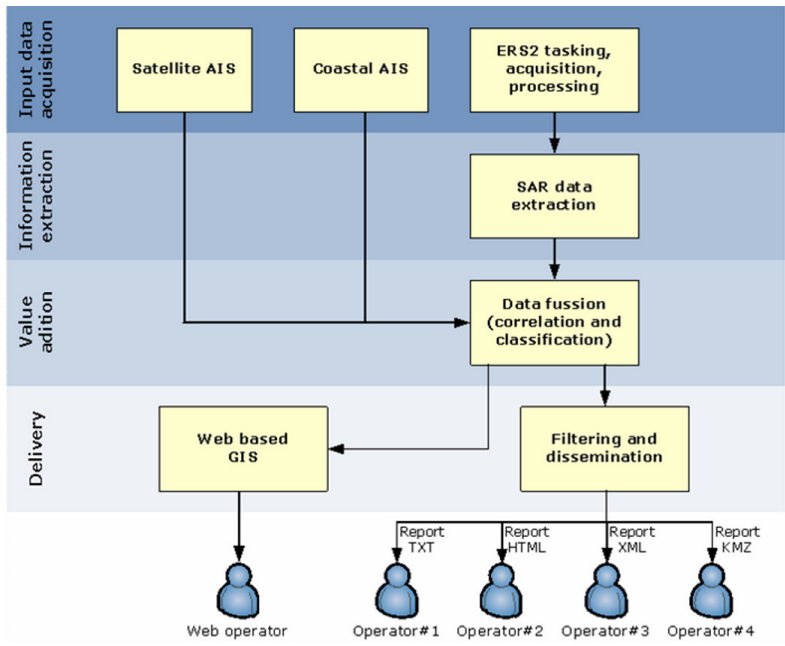

Figure 1: High level scheme of the system. Processing flow is from up to down. 
signatures. Ship categories come from standard vessel categorizations and their assignment to the located ships is performed via fuzzy logic.

- Product generation and delivery. This stage generates and delivers final products to users. On the one hand, the GeoTIFF format is mainly adopted for output images, whereas .xml and plain text are used for generating the reports. On the other hand, data visualization is performed with an advanced web interface, which is supported by a Geographic Information System (GIS) platform. There, different information layers are added according to user needs, such as geo-coded reflectivity images, SAR- and AIS-based ship locations or added-value products.

Important advantages of SASHIM are its modularity, robustness and friendlyuser interface. In addition, the different stages use state-of-the-art technologies, both for SAR data processing and the web interface. For SAR ship detection and classification, confidence values are provided according to the certainty acquired in generating outputs. This helps to improve added-value products and to make system customization more easy and efficient.

SASHIM has been programmed in Java and SIDECAR in Matlab ${ }^{\circledR}$. Thanks to the Matlab ${ }^{\circledR}$ C-compiler utility, a SIDECAR stand-along version exists. A set of external files are needed to run the system, i.e data reading libraries, shape files for coastline isolation and a text file for defining ship categories. The processing time is competitive, meeting the requirements for near-real time product delivery. It is worth noting that the system is totally automatic and unsupervised. This means that from data ingestion to product delivery no supervision from the operator is performed. System interaction is only performed when the operator defines the user's profile or the preferences for data processing and information layers.

System usefulness has been assessed by cooperation with the Spanish police authorities (Guardia Civil) and naval army. Right now, a version of SASHIM is running in their facilities supporting decision making with a performance that meets quality standards.

\section{SAR image processing}

This section describes the main steps followed by SIDECAR to process SAR images in the scope of ship monitoring. Four main steps are defined, namely: land masking, ship detection, ship classification and output generation. Important information for image processing, such as geo-coding, processing quality parameters, antenna pointing information or space-borne state vectors are assumed to be included in the input meta-data.

\subsection{Land masking}

Land masking is the first step performed after reading input images with the external libraries. Two operating modes are admitted: shape and image. The first mode relies on the usage of shape files, which limit land extension via collections of latitude and longitude pairs. The higher the number of points, the 
higher the accuracy in isolating the sea from the land. Right now, SIDECAR has world coastline files in medium resolution and Spanish coastline files in very fine resolution. Due to the coordinate system of shape files, they do not normally meet image geo-coding information, so system transformations are required. With this mode, coastline isolation is possible with an efficient processing load. However, some inaccuracies may appear for small islands or irregular shorelines, which may be solved with finer shape files.

In the second mode, image processing techniques are used for land masking, but at the expense of increasing processing time. In the current version, the particular reflectivity properties of both types of targets are exploited by combining a gradient variance analysis of RCS with a morphological processing. For intense image distortions or high sea reflectivity, this approach suffers some limitations. However, they can be overcome with the support of shape files. Actually, the algorithm normally operates by combining both operating modes with a safety margin to reduce the impact of inaccuracies.

\subsection{Ship detection}

Ship detection is performed with a variant of the methodology outlined in [7]. There, wavelet transform is used to analyze input image with a multi-scale approach. Qualitatively, multi-scale means to "decompose" the image into basic features by using different and non-periodic "resolution" ratios (or scales) between the spatial and wavenumber domain. The Fourier Transform is a particular case of previous property for which time and frequency dimension has a particular resolution ratio. With wavelets, more than one resolution ratio is considered in a common mathematical framework for image feature analysis. Behind wavelet there is a vast theory accurately defining the mathematical properties of this signal transformation [8].

In the case of images, there is a more suited variant called Stationary Wavelet Transform 2D (SWT2), which permits to extend the concept of periodic pattern recognition in 1-D signal analysis to the features of 2-D images. In that way, advanced morphological analysis becomes available for the isolation of different features according to the patterns they show along the different scale products. This is the case of ships that presents a marked "coherent" response along wavelet scales, in contrast of what observed for the sea, with a clear noise-like "incoherent" behavior [7]. Thus, ships can be isolated from the sea by applying a pixel-bypixel multiplication along the scale products. The result is a clear enhancement of wavelet-coherent features (ships) with respect to the incoherent ones (sea). This is the casuistic behind the method in [7]. The advantage is that no fine thresholding is required as the dynamic range between ship and sea responses increases a lot. In addition, the processing approach becomes extremely simple as it mostly reduces to a simple pixel-by-pixel multiplication. Then, the binary decision (to decide if a pixel belongs to a ship) is made by a simple feature masking done via histogram followed by the inverse wavelet transform. 
The powerfulness of this approach has been proved with different tests done with real images (see [7]). For such reason, SIDECAR has adopted this methodology, but adding other features more focused to the operational case. First of all, ships are located by doing particular combinations of the different sub-products at the first scale. When the inverse wavelet transform is applied to such combinations, the resulting image highlights ship presence by a collection of close local maxima. An iterative clustering process around the highest local maxima permits then to find the actual ship position. As such, this product offers two advantages, namely:

1. Proximity. The iterative and automatically adaptive clustering procedure helps to locate close ships. Cluster and local maxima thresholds are modified according to the characteristics of image features. In [7], the wavelet scale up to which the input image is processed fixes the resolution for differentiating ships. So, the higher the analyzed scale, the worse the ship location resolution, but the higher the capability to detect small and less dispersive targets. In SIDECAR, the adopted approach tries to overcome this trade-off with an user-defined parameter that fixes the minimum distance to distinguish two close ships.

2. Confidence. According to the characteristics of local maxima, a confidence parameter $\mathrm{C}$ indicating the percentage of certainty that the algorithm has in labelling a detected spot as a ship can be defined. The experience gained with the analysis of several images shows that specific range of values of $\mathrm{C}$ indicates the presence of particular image features. For instance, low confidence $(\mathrm{C}<0.3)$ is indicative of ship-like sea aberrations, such wave crests; medium confidence $(0.3<C<0.6)$ of small ships with low dispersion (potential clandestine boats); and finally, high confidence ( $\mathrm{C}>$ 0.6) of medium and large high-dispersive ships, which can be easily located by eye inspection. $\mathrm{C}$ has been found to be very useful for supporting the definition of classification, better decision making and efficient alarms.

Fig. 2 shows an example of independent coast isolation and ship detection carried out by SIDECAR for an ESA'S ENVISAT image sensing the surroundings of Gibraltar, Spain, in Image Precision Mode. As observed, coast isolation is accurate and ship detection performs very well. Certainly, not only the ships identified by eye inspection are located, but also other ones with low RCS. Specially interesting are the two spots cruising from South to North and marked with white rectangles. The associated features and the confidence assigned by the system make them potential candidates to be clandestine boats boarding illegal immigrants on. But without ground-truth such asseveration is difficult to formulate.

\subsection{Ship classification}

Ship classification lays on analyzing the distribution of RCS along the signature isolated in SAR image. Signature isolation is performed by applying an iterative gradient variance analysis for which cluster of pixels with similar reflectivity 

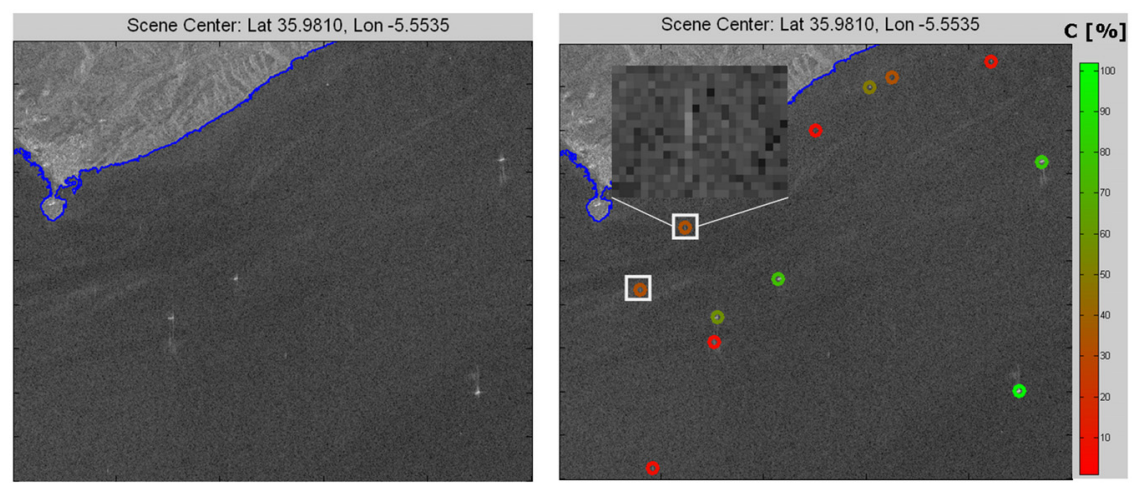

Figure 2: Snapshots for independent coastline isolation (left) and ship detection (right). The imaged area is about $164 \mathrm{~km}^{2}$ and belongs to an ESA'S ENVISAT imaged acquired at 27-12-2007 for the surroundings of the Strait of Gibraltar with a resolution of $30 \mathrm{~m}$. Pixel spacing is $12.5 \mathrm{~m}$.

properties are inter-connected. Adaptive thresholds define ending criteria and discard pixel clusters with no signature relation.

Once the ship signature is isolated, the heading is estimated via the Radon Transform [9]. Qualitatively, this mathematical tool permits to project the image into a set of oriented lines and calculate the related correlation coefficient. Thus, for a linear feature, the highest coefficients indicate the preferred orientation (see Fig. 3). In order to increase accuracy, an adaptive clustering approach similar to that used for ship detection has been adopted. With heading information, ship dimensions are then estimated by simply counting the pixels along the bow-stern and board-starboard axis.

The next step is to evaluate RCS distribution along ship signature. For such purpose, three sections with almost the same area are defined: bow, middle and stern. For each one, a mean value is retrieved, which is normalized to the maximum value of the signature (other coefficients related with image quality and sea statistics are used). Fuzzy logic is then applied to find which category from the reference ones better adapts to the dispersive and geometrical characteristics of the isolated target. Table 1 shows a summary of the accounted categories as defined in the classification reference file.

In simple words, fuzzy logic is the theory for which classical binary levels are extended to a continuum grades of intermediate memberships [10]. So, the conventional crisp of full- and non-membership levels ( 0 and 1 ) is overcome to handle imprecise information. The number of levels are known as grades, which are defined by membership functions. Such functions provide the percentage that an input is a member of each grade. The number of grades, the shape of membership functions and the percentage of overlap is flexible and updated according to the application to develop. They conform what is know as fuzzy 


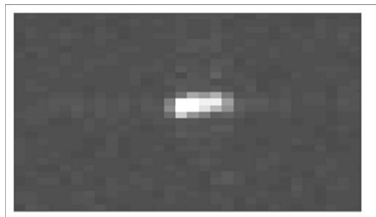

ORIGINAL IMAGE

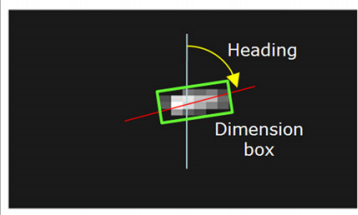

ORIENTATION AND DIMENSIONS

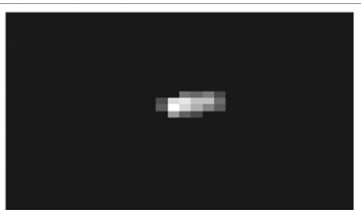

SIGNATURE ISOLATION

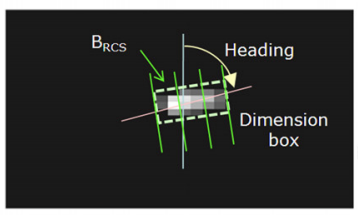

SECTION SIGNATURE DIVISION

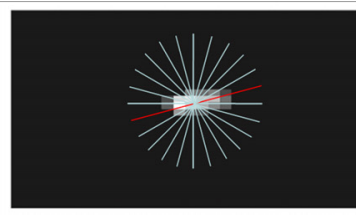

ILLUSTRATIVE RADON TRANSFORM

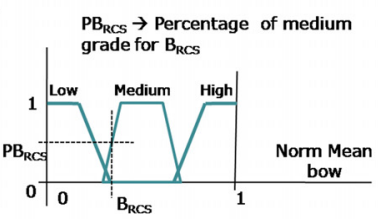

MEMBERSHIP FUNCTION FOR MEAN BOW

Figure 3: Illustrative description of the different steps followed in ship classification without taking fuzzy logic processing into account.

Table 1: Summary of ship categories with the grades they have for the three normalized RCS values. Typical ship dimensions values are also included. This info is used to classify ship signatures by combining fuzzy-based "ifthen" statements.

\begin{tabular}{|c|c|c|c|c|c|}
\hline Category & $S_{R C S}$ & $M_{R C S}$ & $B_{R C S}$ & Length [m] & Breadth [m] \\
\hline Oil Tanker & High & Low & Low & 300 & 45 \\
\hline Chemical & High & Low & Medium & 200 & 35 \\
\hline Coaster & High & Medium & Low & 100 & 25 \\
\hline Goods Cargo & High & Low & Medium & 150 & 25 \\
\hline Gas Cargo & High & High & Low & 200 & 35 \\
\hline Bulk & High & Medium & Low & 250 & 35 \\
\hline Container & High & High & High & 225 & 45 \\
\hline Car Ferry & Medium & High & Low & 150 & 25 \\
\hline Fishing vessel & Medium & High & Low & 75 & 25 \\
\hline Small targets & Low & Medium & Low & 15 & 5 \\
\hline
\end{tabular}

"set". More than one parameter can be evaluated by fuzzy rules, which outputs are normally combined by "if-then" statements.

In the case of SIDECAR, the normalized RCS of bow $\left(B_{R C S}\right)$, middle $\left(M_{R C S}\right)$ and stern $\left(S_{R C S}\right)$ sections are the input fuzzy parameters. Three grades are considered (low, medium and high) with the membership functions shown in Fig. 3 . For each category, a particular grade combination for $B_{R C S}, M_{R C S}, S_{R C S}$ is defined according to experience (see Table 1). 
For evaluating the percentage that a measured ship signature is member of the different reference categories, two steps are followed. In the first one, the percentages of $B_{R C S}, M_{R C S}, S_{R C S}$ to pertain to the grades of each category are calculated (see Table 1). These values are then evaluated with the set of "if-then" statements that combines the associations in Table 1 (ship dimensions are also accounted for). In the second step, a two-grade fuzzy "set" ("false" $\rightarrow$ no category member and "true" $\rightarrow$ category member) is defined with symmetric membership functions. The output of "if-then" statements is used to evaluate the percentage that the processed category is member of the "true" and "false" grade. Both percentages permits to define an area under the "true" and "false" membership functions, which becomes the final similarity value between the measured ship signature and the processed category. The highest similarity identifies the category assigned to the measured ship signature, which has an associated confidence value retrieved directly from fuzzy processing.

\subsection{Output}

SIDECAR generates two output main files, namely: a) a GeoTIFF reflectivity image with the geo-coding information of input meta-data and b) a report in .$x m l$ and plain text summarizing processing results. Included information is ship geographic location and category, detection and classification confidence, heading, dimensions and $B_{R C S}, M_{R C S}, S_{R C S}$.

\section{Web interface}

Despite SIDECAR has been designed to easily integrate the results into other systems, a dedicated web interface (http://mariss.gmv.eshttp://mariss.gmv.es) has been implemented with state-of-the-art web technologies. The interface shows the processing results in a simple and handy way, through a GIS implemented with open source tools (as MapServer and OpenLayers) and using geographic standards (as WMS and WFS) (see Fig. 4).

Some different dissemination strategies can be configured with diverse formats (plain text, XML, PDF, Google Earth KMZ, etc.). The reports that are automatically generated after receiving SAR products can be easily distributed to different operators or integrated in different platforms. The interface also allows users to configure filters in order to reduce the information to report, or the way it is displayed. Thus, operators can focus only on their areas of interest, or on detections that activate certain alarms.

\subsection{SAR-AIS correlation}

Another important web feature is the correlation between SAR and AIS ship detections. The process starts by gathering the AIS information over the same location and period covered by the SAR image. All the detections for the same vessels are grouped into route tracks, and their expected locations into the SAR 
334 Safety and Security Engineering III

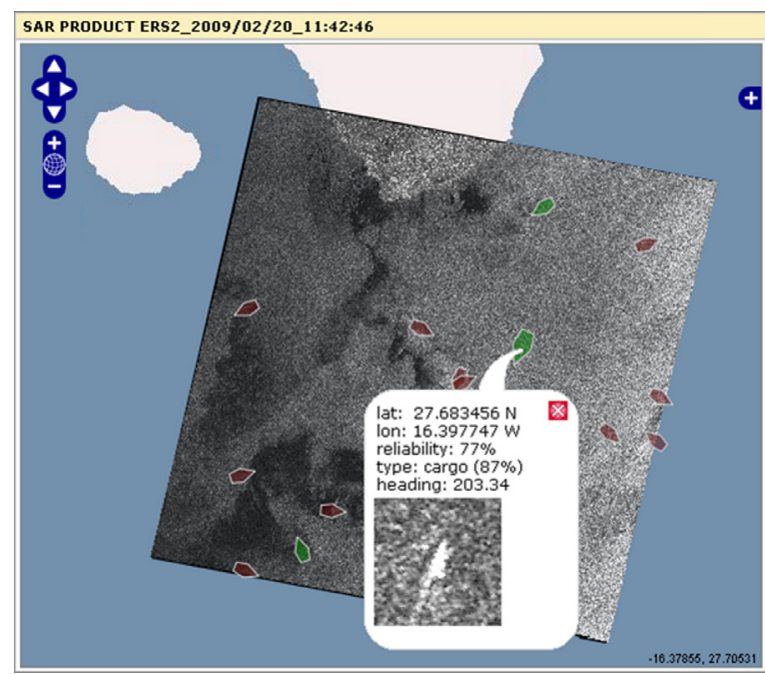

Figure 4: SASHIM GIS support for an ERS image of the Canary Islands.

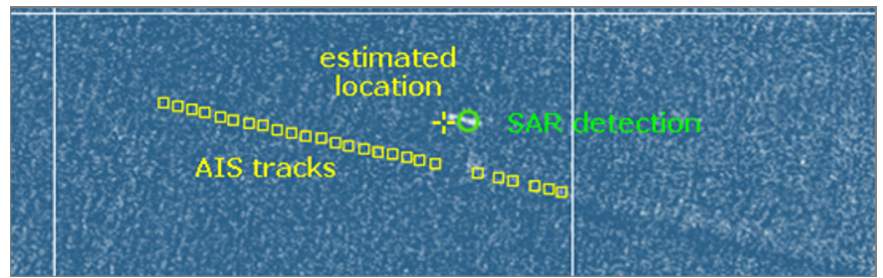

Figure 5: Illustrative description of the AIS-SAR route tracking algorithm.

image calculated at image acquisition time (see Fig. 5). This process accounts two undesired effects, which are compensated with a route propagation algorithm, namely: 1) ship location shifts in SAR images due to Doppler effects and 2) low number of AIS tracks. When an AIS ship position is close enough to a SAR detection, then a positive match is labelled and a confidence value calculated. This parameter depends on the relative distance between SAR and AIS ship location, on ship dimensions and heading.

\section{Results and AIS cross-correlation}

A lot of medium resolution (30 m) ERS and ENVISAT images covering different areas (Strait of Gibraltar, Mauritania, Canary and Azores Islands ...) have been used to test SASHIM performance in autonomous mode. This section will provide a brief summary of the most significant results. First of all, Fig. 6 presents a snapshot of the SASHIM GIS support for an ERS image acquired for the Strait of Gibraltar. There, left image shows the detections performed by SIDECAR with 

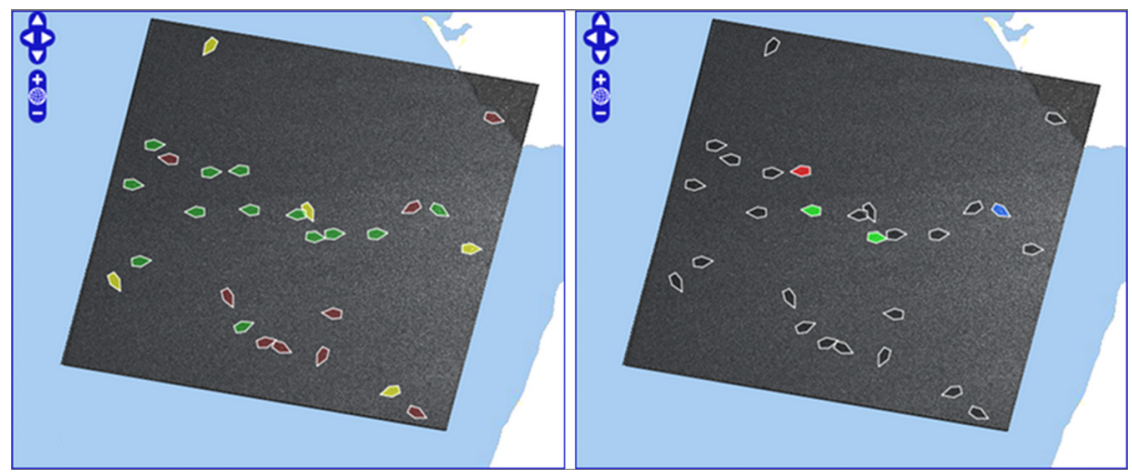

Figure 6: Examples of SASHIM outputs for an ERS image of Mauritania.

a color-code indicating the level of confidence, whereas right image the result of classification. There, the color-code indicates the assigned category with red for oil tanker, green for container, blue for goods cargo and black for unclassified.

The results show that ship detection performs very well as not only all the ships that can be identified by eye inspection are located, but also other ones less evident. Note that system output permit to see at a glance where the ships are and which course they are following. In addition, color code helps to clearly identify detection confidence (green C > $70 \%$, yellow $70 \%>\mathrm{C}>40 \%$, red $40 \%>\mathrm{C}$ ) and the assigned category according to user profile and the configured alarms.

Another important item is to show how ship classification performs. For such purpose, ship detections have been cross-correlated with AIS polls to see the matching performance of the assigned categories. With previous AIS-SAR correlation procedure, a test has been carried out for the ENVISAT image partially shown in Fig. 2. Table 2 summarizes the cross-correlation of categories for the AIS-SAR ship correlations having the highest confidence. The results show that the category assigned by the algorithm matches the category of the observed vessel in around 55-60\%. This result can be considered good for the type of managed images, i.e. medium resolution single-polarimetric ENVISAT and ERS images.

\section{Conclusions}

This paper has presented an operative ship monitoring approach that combines the information obtained from AIS polls with SAR image processing. A stateof-the-art web interface supports output presentation with the aim to increase user interaction and added-value capabilities, such alarm generation, user profile definition of ship classification.

Some tests have shown the good performance reached by SIDECAR and, in general, by SASHIM. Proper land masking, accurate ship detection and reliable ship classification has been found for an algorithm that meets near-real time requirements. In addition, it is fully operative, robust and unsupervised. The 
Table 2: Summary of AIS-SAR correlation applied to an ENVISAT image for evaluating ship classification performance. Bold labels indicate positive matching.

\begin{tabular}{|c|c|c|c|c|}
\hline Category SAR & Coaster & Oil Tanker & Bulk & Goods Cargo \\
\hline Category AIS & Fast-Ferry & Container & Bulk & Oil Tanker \\
\hline Ship Name AIS & Avemar dos & MSC Levina & Maersk Nagoya & Hekabe \\
\hline Category SAR & Oil Tanker & - & - & - \\
\hline Category AIS & Oil Tanker & - & - & - \\
\hline Ship Name AIS & Omega Theodore & - & - & - \\
\hline
\end{tabular}

operator only has to interpret the results taking profit of the large amount of ancillary data provided by the system. User feed-back has shown that the system is very useful for supporting decision making in the field of immigration control and ship activities. For such reason, some upgrades have been asked for future research, namely:

- Automatic Coastline detection with the wavelet-based method of [11]. An unsupervised behavior without the support of shape files is demanded.

- To devote more research to increase the capability to detect small and less dispersive targets for immigration activities.

- To improve Ship classification by revising ship categories and the associated fuzzy "sets". This implies to evaluate other membership functions, grade distribution and additional input fuzzy parameters.

- To generate a database of detection and classification collections for developing Auto-learning algorithms based on neural networks. They may be used to update, if required, fuzzy classification configuration.

- To develop other Added-value capabilities, such as ship tracking along time. Stacks of SAR images or AIS-SAR correlation may be used.

- To develop more Tests with the datasets of new sensors such TerraSAR$\mathrm{X}$ or Cosmo-Skymed. There, the improved resolutions and polarimetric capabilities may cause to adapt ship detection and classification.

\section{References}

[1] International Maritime Organization (IMO), United Nations (UN), Automatic Identification System (AIS), [Online] Available at: http://www.imo.org/includes/blastDataOnly.asp/datajd\%D6645/227.pdfI\% MO web page.

[2] Cumming, I.G. \& Hong, F.H., Digital processing of Synthetic Aperture Radar. Artech House, INC.: Norwood, MA, USA, 2005.

[3] ESA, MARISS project [Online]. Available at: http://www.gmesmariss.com/MARISS Web page. 
[4] EC., VMS: [Online] Available at: http://ec.europa.en/fisheries/cfp/control_ enforcements/vms_en.htmVMS web page.

[5] Margarit, G., Mallorqui, J.J. \& Fabregas, X., Single-pass polarimetric SAR interferometry for vessel classification. IEEE Trans Geosci Remote Sensing, 45(11), pp. 3494-3502, 2007.

[6] Margarit, G. \& Mallorqui, J.J., Scattering-based model of the SAR signatures of complex targets for classification applications. Intern Journal of Navi and Obser, Special Issue on Modelling and Processing of Radar Signals for EO, 2008(ID: 426267), p. 11 pages, 2008.

[7] Tello, M., Lopez-Martinez, C. \& Mallorqui, J., A novel algorithm for ship detection in SAR imagery based on the wavelet transform. IEEE Geosci Remote Sensing Letters, 2(2), pp. 201-205, 2005.

[8] Mallat, S.A., Tour of Signal Processing. Academic Press Inc., 1998.

[9] Wehner, D.R., High Resolution Radar. Artech House: Boston, 1995.

[10] Tanaka, K., An Introduction to Fuzzy Logic for Practical Applications. Rassel, Inc.: New York, USA, 2007.

[11] Tello, M. \& et. al, Automatic detection of spots and extraction of frontiers in sar images by means of the wavelet transform: Application to ship and coastline detection. Proc. IEEE International Geoscience and Remote Sensing Symposium (IGARSS'06), pp. 383-386, 2006. 